\title{
LASER DIODE DISTANCE MEASURING INTERFEROMETER - METROLOGICAL PROPERTIES
}

\section{Marek Dobosz}

Warsaw University of Technology, Faculty of Mechatronics, Institute of Metrology and Biomedical Engineering, Św. A. Boboli 8, 02-525 Warsaw,Poland( $\triangle$ dobosz@mchtr.pw.edu.pl, +48 22234 8347)

\begin{abstract}
A novel laser diode based length measuring interferometer for scientific and industrial metrology is presented. Wavelength the stabilization system applied in the interferometer is based on the optical wedge interferometer. Main components of the interferometer such as: laser diode stabilization assembly, photodetection system, measuring software, air parameters compensator and base optical assemblies are described. Metrological properties of the device such as resolution, measuring range, repeatability and accuracy are characterized.
\end{abstract}

Keywords: diode laser, wavelength stabilization, interferometer, interferometer distance measurement.

(C) 2012 Polish Academy of Sciences. All rights reserved

\section{Introduction}

When visible diode lasers came out many researches often wondered about their suitability as a replacement for the HeNe. HeNe lasers are rather bulky, sensitive to external vibration and magnetic fields. They require high voltage power supplies. They are also a major heat radiation source. On the other hand coherence length is determined by the bandwidth of the laser, which in most cases is related to cavity length. Since this is only a fraction of a millimetre in laser diodes, the coherence length is often very short. However laser diodes designers have come up with many sorts of ways of getting a narrow bandwidth from a short cavity. Some standard (i.e. very cheap) laser diodes now have coherence lengths approaching several meters. Applying additional optical components such as internal or external filters, might push the coherence length to tens of meters. However in this case the complexity of the $\mathrm{LD}$ increases and the price of the device reaches or exceeds the price of $\mathrm{HeNe}$ lasers.

Since coherence lengths of the LDs increased significantly, lasers of this kind are becoming increasingly important in length metrology, especially in interferometric techniques. The wavelength of a diode laser is determined primarily by the band gap of the semiconductor material though there is also strong dependence on diode temperature and current density. This way the frequency stability of visible diode lasers, used as frequency standard in a laser interferometer, has to be improved by applying an active system of laser frequency stabilization.

There are many interferometer systems based on a laser diode source of light. However the majority of them till now are experimental systems dedicated to special type of measurement. The most classic approach to the problem of interferometer distance measurement can be found in e.g. [1, 2]. Recently G. Dudzik et all [3, 4] developed a VCSEL laser diode stabilized using absorption spectroscopy and rubidium vapour cell. He has shown that it is possible to apply a laser stabilized in this way for a length-measuring interferometer but a metrological 
analysis of this system has not been carried out. Also the infrared range of the applied laser will cause many problems in an attempt to develop a useful interferometer.

Examples of more specialized interferometer systems based on laser diode properties are shown in [5-9]. An example of applying laser diode feedback interferometry is shown in [5]. The basis of sinusoidal phase-modulating laser diode interferometry has been given by $\mathrm{O}$. Sasaki et al. [6]. Examples of applying a photothermal LD modulating technique were recently presented by X. Wang et al. [7]. An example of LD phase-conjugate interferometer for distance measurement has been presented by Ishii and Takahashi [8]. Finally a semiconductor miniature chip LD-based interferometer is offered by [9].

Many LD frequency stabilization methods were reported. A survey of the existing systems can be found for example in the following references [10-14]). The majority of them are complex and expensive. The idea of a simple laser wavelength stabilization system based on the wedge interferometer has been proposed by Alexander A. Zhmud [15]. A very similar technique was applied by J. C. Brasunas [16], employing an uncoated etalon to monitor the laser wavelength and then applying a correcting heating term to the laser to stabilize its wavelength in a varying temperature environment.

We developed the idea of applying an uncoated glass etalon as a reference interferometer for LD frequency stabilization [17, 18].The proposed system uses an interference pattern from the external wedge etalon to detect wavelength (frequency) changes via a differential photodetector. An electronic circuit controls the LD current in the feedback loop so as to obtain the LD wavelength as close as possible to the value set in the calibration procedure.

A more detailed description of the proposed technique has been presented in $[17,18]$. Basing on this stabilization method we have developed a small and compact, inexpensive laser diode based interferometer similar in general design to standard commercially available HeNe laser interferometers. We demonstrate herewith especially novel elements of the design and construction of the interferometer. Experimental verification of the main metrological properties of the device such as resolution, measuring range repeatability and accuracy is shown.

\section{Laser head}

The laser head of our new laser diode based distance measuring interferometer fulfils two basic functions:

- emission of a laser beam having the required properties like the coherence length, frequency stability, beam diameter etc.,

- reception of the returning beams (measuring and reference) and processing the resulting interference into output signals that are in quadrature.

\subsection{LD wavelength stabilization accuracy}

Wavelength instability and reproducibility of the LD was tested by comparison of the instantaneous values of the LD wavelength with the wavelength of a reference stabilized HeNe laser in vacuum. For this purpose we used a specially designed interferometric vacuum wavelength comparator $[17,18]$. The measurement relative uncertainty of the comparator was equal to about $3 \times 10^{-9}$ (range). The frequency instability of the applied reference HeNe laser was about $\pm 1 \times 10^{-9} / \mathrm{h}$. Instability of the LD frequency was measured in 8 -hour periods of time.

In metrology applications Allan deviation is a popular measure of laser frequency instability [19]. Table 1 shows exemplary values of Allan deviation obtained in 3 measurement series for different time of averaging [17]. 
Wavelength reproducibility is as much as instability important in interferometric distance measurements. We tested this parameter using the method described earlier i.e. by comparison of the LD wavelength with the HeNe reference wavelength by means of an interferometer operating in vacuum. During the test the LD head was turned on and off with 30 min brakes. The HeNe laser was operating continuously. After 30 min preheat time of the LD head, a 10 min measurement of the wavelength was performed. For each $10 \mathrm{~min}$ period of time averaging the LD wavelength was calculated. Observed differences between these averages can act as wavelength reproducibility. In repeated measurement series we have obtained relative wavelength changes not exceeding $5 \times 10^{-8}$.

Table 1. Exemplary values of Allan deviation obtained in 3 measurement series.

\begin{tabular}{|c|c|c|c|}
\hline \multirow{2}{*}{ Measuring series } & \multicolumn{3}{|c|}{ ALLAN deviation } \\
\cline { 2 - 4 } & \multicolumn{3}{|c|}{ Averaging time [s] } \\
\cline { 2 - 4 } & 0 (No averaging) & 1 & 10 \\
\hline Series 1 & $2.8^{-8}$ & $5.7^{-10}$ & $5.7^{-11}$ \\
\hline Series 2 & $6.6^{-8}$ & $1.3^{-9}$ & $1.3^{-10}$ \\
\hline Series 3 & $7.7^{-8}$ & $1.4^{-9}$ & $1.4^{-10}$ \\
\hline
\end{tabular}

Assuming the applied thermistor (used for etalon stabilization) drift generates relative wavelength instability at the level of $2 \times 10^{-8} /$ year, we can expect the overall LD head wavelength reproducibility at the level of about $10^{-7}$ /year.

\subsection{Fringe detection system}

In each kind of distance measuring interferometer based on the counting fringe technique, a photodetection system that can converts the interference signal into two $\pm 90^{\circ}$ phase shifted output signals is necessary. The sign of this phase shift indicates the measured displacement direction. The phase shifted output signals also allow to increase the measurement resolution by interpolation techniques. For the purpose of our LD interferometer construction we have developed a novel photodetection system based on analyzing fringes of finite period by means of a compact size integrated photodetector comprising 13 appropriately connected photoelements. The proposed novel method of obtaining sine and cosine output signals is described by following equations:

$$
\begin{gathered}
I_{I}=\frac{\left(I_{1}+I_{5}+I_{9}+I_{13}\right)}{2}-I_{3}-I_{11}=4 \sin \alpha, \\
I_{I I}=I_{2}-I_{4}+I_{10}-I_{12}=4 \cos \alpha .
\end{gathered}
$$

It is easy to notice that the above signals do not have a DC component. The applied system assures a very high accurate $90^{\circ}$ phase shift between measuring signals, reducing distance measuring errors. The photodetection system has been described in detail in [17, 20-22]. 


\subsection{Opto-mechanical design of the system}

A general view of the laser diode interference head is shown in Fig. 1 [17]. The optomechanical part of the laser head consists of two fixed together and thermally stabilized following blocks: (i) laser diode assembly and (ii) etalon assembly i.e. the optical wedge interferometer used for laser frequency stabilization. Both LD and the etalon are mounted inside separate copper bodies. These units are thermally isolated from the external housing by ceramic distances. Temperature of the LD is precisely controlled by a thermoelectric cooler (TEC) while the etalon temperature is stabilized with higher accuracy by keeping it at a fixed temperature.

The laser output beam is formed into a circular shape by means of an anamorphic beam expander. The HL6312G (Hitachi) LD has been used in our system.

Fig. 1(a) shows the internal view of the prototype device. Two blocks of LD and etalon sets fixed together are visible. In front of them the anamorphic prisms set is visible. A general view of the LD interferometer head is shown in Fig. 1(b).

a)

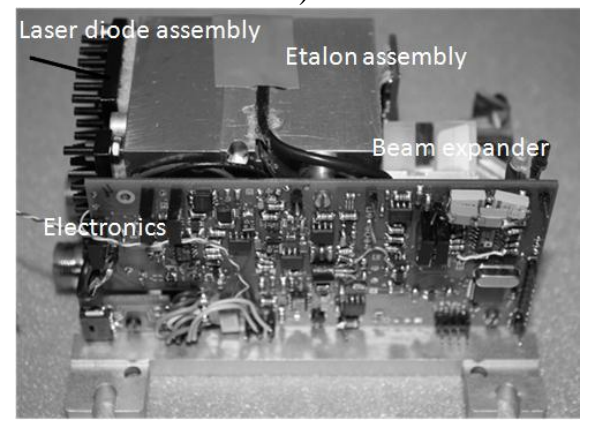

b)

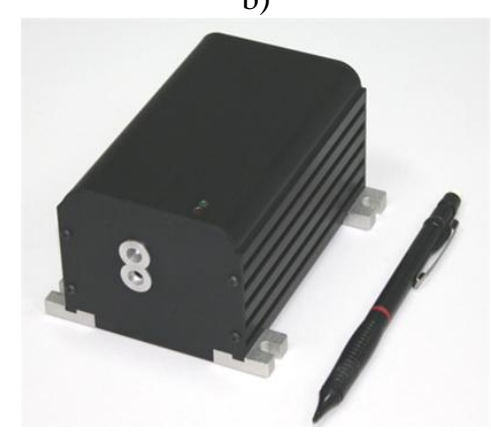

Fig. 1. LD interferometer head. a) internal b) general view of the head.

\section{Fringe counting and interpolation}

The fringe counting and interpolation circuit has been designed as a separate assembly that can be used not only for the described interferometer signal processing but also in other applications. It is an intermediary assembly between the laser head and the computer. The communication of the device with the external computer is realized by a USB port. The counting and interpolation circuit design is based on an ADUC841 processor that contains built-in 12-bit analog-to-digital (A/D) converters.

The sine and cosine signals outgoing from the photodetector system are digitized by A/D converters. The most significant bit outputs follow the zero crossings of the signals. These are the digital quadrature signals that are used to drive a reversible counter. The instantaneous interpolated interference phase angle $\alpha$, is calculated from:

$$
\alpha=\arctan \left(I_{I} / I_{I I}\right)
$$

where: $I_{I} ; I_{I I}$ are sine and cosine signals. In order to make this calculation as accurate as possible the described circuit allows for programmable precise adjustment of the zero value of the DC part of these signals.

The sine and cosine signals readings can be triggered by a $1 \mathrm{kHz}$ internal clock or by an external pulse. The signal readings are stored in a fast internal memory that has in the current 
version a capacity of 4096 samples, or they can be sent via a USB port to the PC memory. The circuit internal settings are stored in its internal EEPROM memory.

The interpolator unit dimensions are $54 \times 84 \times 24 \mathrm{~mm}$.

A detailed description of the counting and interpolation circuit is presented in [17].

\section{Software dedicated to the LD interferometer}

We have developed a universal software that can handle any laser interferometer from which the measurement data are sent to a PC in a fixed format. The software package includes standard modules for linear, angular measurements including velocities and acceleration measurements. Modules for vibration analysis and coordinate measuring machine testing are available also.

This software has been especially dedicated to the LD based interferometer. The most important features that dedicate this application to the LD are:

- Ciddor's-formula-based correction of the applied laser wavelength. It is especially important in the case of a laser diode that emits a wavelength differing from the $633 \mathrm{~nm}$ standard HeNe laser,

- The procedure of the wavelength correction that takes into account the thermal changes of the laser frequency.

The developed software also has several features that one will not find in a standard commercially available laser interferometer. The most important include:

- Computer-aided adjustment of the quadrature signal phases,

- A quadrature interference signal phase error numerical compensation algorithm especially useful in sub-nanometer resolution measurements.

Computer aided adjustment of the quadrature signal phase software window is shown in Fig. 2. We can observe in this window a Lissajous figure generated by the actual sine and cosine signals (visible dots). Arrows visible on the left side allow to adjust signal offsets to match the measuring signal to the reference circle (full line).

a)

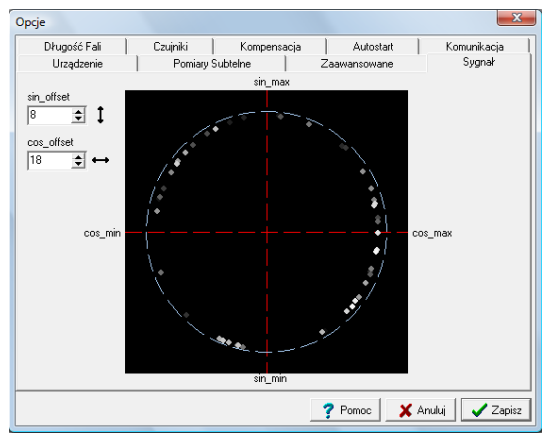

b)

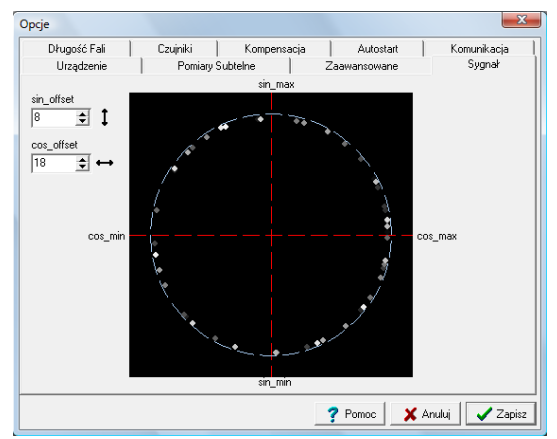

Fig. 2. Preview window of the interference signal quality. a) Lissajous figure generated by the signals disturbed a small phase error. b) the same Lissajous figure after phase error numerical compensation.

Vibrations or small test movement of the interferometer reflector can deliver to the software a measuring data for the purpose of interference signal phase error evaluation (i.e. difference between actual signal phase shift and $90^{\circ}$ ). Basing on this information a procedure of numerical phase error compensation algorithm is implemented. Fig. 2a shows a small deformation of the Lissajous figure generated by the measuring signals. It is obviously caused by the signal phase error. Fig. $2 b$ shows the same figure after this error numerical compensation. A detailed description of the developed software has been presented in [17]. 


\section{New approach to air conditions compensator design}

Air conditions compensation is a key factor in each interferometer system accuracy. The air conditions compensator very accurately measures air temperature, air pressure and relative humidity. It then modifies the nominal value of the laser wavelength to give a true value, used in calculations as a length master.

Up to now analog air sensors are used as most accurate devices. However transferring measuring information from a sensor to the electronic circuit via cable and connectors is always a source of errors.

In order to reduce the cost of the compensator construction we decided to apply in our interferometer system only integrated sensors that give the output directly in a digital form. Since the digital sensors are not so accurate as traditional analog devices we decided to implement in each case the digital compensation of the measurement error inherent to the each sensor.

The developed wavelength compensator is designed in the form of an independent device that can measure air parameters and send them via the USB port to any PC that is equipped with a simple software.

Below information is given about types of the applied digital sensors:

- temperature measurement : Maxim-Dallas DS1631,

- pressure measurement: VTI SCP1000 D01,

- relative humidity measurement: Sensirion SHR-11.

As an example of applied numerical compensation of the sensor's systematic errors we show below the method of the temperature reading correction. The original Maxim-Dallas DS1631 error curves of three exemplary items measured in our laboratory are shown in Fig. 3a. In the range from about $10^{\circ} \mathrm{C}$ to $40^{\circ} \mathrm{C}$ the nonlinearity of the tested sensors reaches $0.5^{\circ} \mathrm{C}$. According to the producer specifications [23] these errors can be described by the quadratic function

$$
E=C+B T_{T S}+A T_{T S}^{2},
$$

where $E$ is measurement error, $T_{T S}$ is the actual temperature i.e. temperature measured by the reference temperature sensor. $A, B, C$ are constant coefficients that can be evaluated for each sensor. Using the multiple regression technique we calculated these coefficients for each

a)

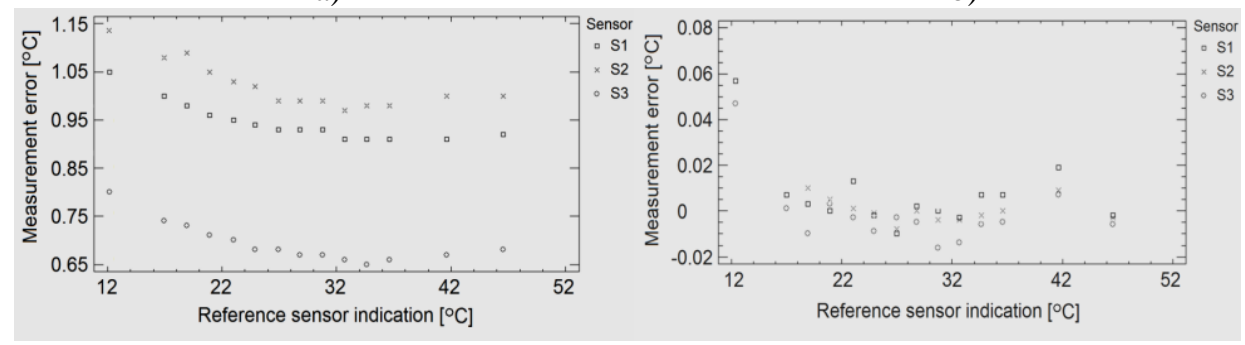

Fig. 3. Measurement errors of three exemplary items of Maxim-Dallas DS1631 temperature sensor. a) before, b) after numerical error compensation.

tested sensor. For example values of $A, B, C$ coefficients obtained for sensor $\mathrm{S} 1$ shown in Fig. 3a are equal to $0.000229286 ;-0.0180554$ and 1.2556 respectively.

Basing on equation (4) and using the evaluated regression coefficients for each sensor the sensor readings can be corrected. The error characteristics of the analyzed sensors after 
numerical correction are shown in Fig. 3b. It is evident that the applied calibration technique reduced temperature measurement errors to the level of $\pm 0.01^{\circ} \mathrm{C}$ in the range interesting us from about $15^{\circ} \mathrm{C}$ to $35^{\circ} \mathrm{C}$. Though the resolution of the sensor is equal to $0.0625^{\circ} \mathrm{C}$, that gives a discretization error equal to $\pm 0.0625^{\circ} \mathrm{C}$; it can be easily limited by readings averaging.

Similarly to the shown temperature sensor calibration, we have reduced errors of the pressure and humidity measurement by applying linear regression and offset correction respectively.

In Table 2 the metrological parameters of the developed air conditions compensator are collected. In the last column of Table 2 the uncertainty of the air refraction coefficient evaluation (thus also the wavelength) is given. The obtained results are very close to accuracies offered by commercially available analog devices.

A detailed description of the developed air condition compensator has been presented in [17].

Table 2. Metrological parameters of the air compensator developed for the LD interferometer.

\begin{tabular}{|c|c|c|c|c|}
\hline $\begin{array}{c}\text { Uncertainty of } \\
\text { measurement }\end{array}$ & Temperature & Pressure & $\begin{array}{c}\text { Relative } \\
\text { humidity }\end{array}$ & $\begin{array}{c}\text { Refraction } \\
\text { coefficient }\end{array}$ \\
\hline Single reading & $\pm 0.1^{\circ} \mathrm{C}$ & $\pm 50 \mathrm{~Pa}$ & $\pm 3 \%$ & $0.174^{*} 10^{-6}$ \\
\hline $\begin{array}{c}\text { Average from } 10 \\
\text { readings }\end{array}$ & $\pm 0.06^{\circ} \mathrm{C}$ & $\pm 50 \mathrm{~Pa}$ & $\pm 3 \%$ & $0.154^{*} 10^{-6}$ \\
\hline
\end{tabular}

\section{Resolution of the interferometer}

Nominal resolution of the presented system resulting from the applied analog to digital converter resolution is equal to about $0.04 \mathrm{~nm}$. However especially external disturbing vibrations that affect the measuring setup do not allow to measure displacements with such a resolution. In order to verify the resolution possible to obtain in our system we developed a measuring interferometer optical setup that is to a large extent insensitive to external disturbing vibrations. The setup is shown in Fig. 4a.

a)

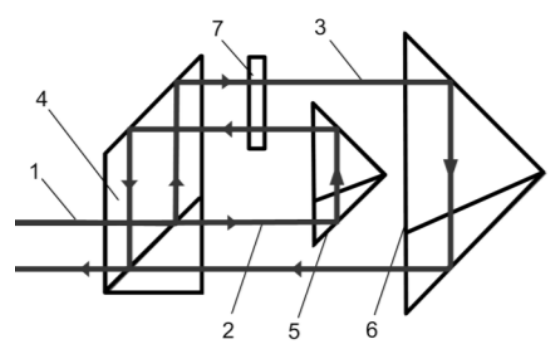

b)

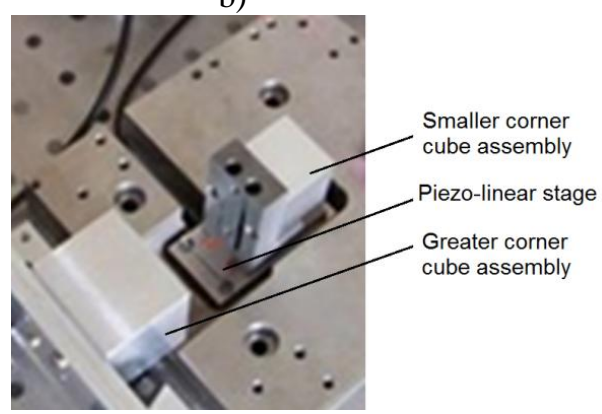

Fig. 4. a) Optical setup for displacement measurement with limited sensitivity to external vibrations. b) View of the optical assembly of the interferometer.

Light beam 1 emitted by the laser head is divided into two mutually coherent beams 2 and 3 by means of a polarizing beam splitter 4 . The beams 2 and 3 have mutually orthogonal linear polarizations and run in perpendicular directions. The beam 2 is reflected parallel to the incidence direction by a fixed, corner cube reflector 6 , while beam 4 is reflected by moving corner cube reflector 5, whose displacements are measured. Of course, reflector 5 can act as the reference reflector while corner cube 6 can act as the measuring reflector. Both beams 2 and 3 pass once by a half retarder plate 7 that turns their transmitted light beam polarization 
by $90^{\circ}$. Due to that beams 2 and 3 recombine after passing again by the polarizing beam splitter 4 and they are analyzed by the photodetection system described in section 2.2.

Since the interfering beams run parallel and both reference and measuring reflector are mounted on the same base, the set-up has limited sensitivity to external vibrations.

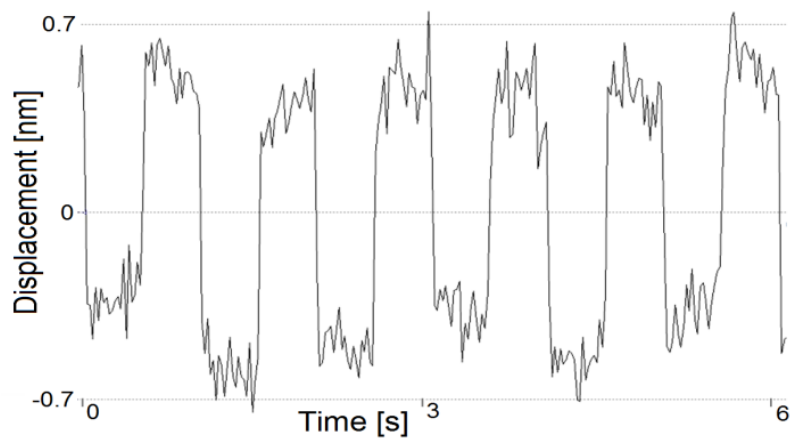

Fig. 5. Approximately $1 \mathrm{~nm}$ (peak to peak) reflector's vibrations measured by the LD interferometer.

Fig. $4 \mathrm{~b}$ shows a view of the measuring set-up. The bigger cube corner reflector 6 was fixed to the base plate while the smaller one (5) was fixed to the nano-positioning piezo-linear stage. We have eliminated the half retarder plate by utilizing polarization effects of the applied cube corners. Using this stage we generated small vibrations of the reflector 5 observing the interferometer indications. Exemplary results are shown in Fig. 5 where the interferometer readings of about $1 \mathrm{~nm}$ (peak to peak) amplitude are shown. The period of the vibrations was equal to $1 \mathrm{~s}$. Each point on the graph represents an average of 200 interferometer readings.

\section{Repeatability and measuring range of the prototype interferometer}

For the purpose of interferometer repeatability and measuring range evaluation, we have used a standard interferometer optical configuration with cube corner reflectors. The experiment evaluated the repeatability of measurement of the interferometer cube corner reflector position for different values of the OPD. In this experiment we tried to catch only the interferometer measurement uncertainty component. The optical path difference was changed from $2 \mathrm{~mm}$ to $7 \mathrm{~m}$ with a $200 \mathrm{~mm}$ step, that is, the measuring reflector was moved from a distance of $1 \mathrm{~mm}$ to $3.5 \mathrm{~m}$ from the beam-splitter that was mounted with the reference reflector. To evaluate the measurement repeatability, 1024 interferometer readings were taken for a period of $1 \mathrm{~s}$. To remove the influence of systematic effects on the measurement, each sample of interferometer readings was statistically analyzed using the following steps:

- Evaluation of the trend line using EWMA smoothing technique (with a constant in the range of 0.005 to 0.01 , depending on the analyzed sample),

- Analysis of the probability distribution of differences between obtained interferometer readings and the trend line,

- Evaluation of the range, standard deviation, and expanded uncertainty of each sample.

The exemplary results obtained for the four characteristic values of the OPD are presented below. Figs 6, 7, 8 and 9 show results obtained for $1 \mathrm{~mm}, 1.5 \mathrm{~m}, 2.5 \mathrm{~m}$ and $3.5 \mathrm{~m}$ distance of the measuring reflector from the beam-splitter respectively. Squares, visible in Figs 6 (a) to 9 (a) represent each individual reading. Grey central lines denote the trend of the observations. Histograms representing residual distribution for each case are shown in Figs 6 (b) to 9 (b). In all the cases the normal probability distribution of the observed differences 
was confirmed by statistical tests with a $95 \%$ confidence level. The horizontal agglomerations of results visible in Figs $6 \mathrm{a}$ are caused by $0.04 \mathrm{~nm}$ interferometer digital resolution.

a)

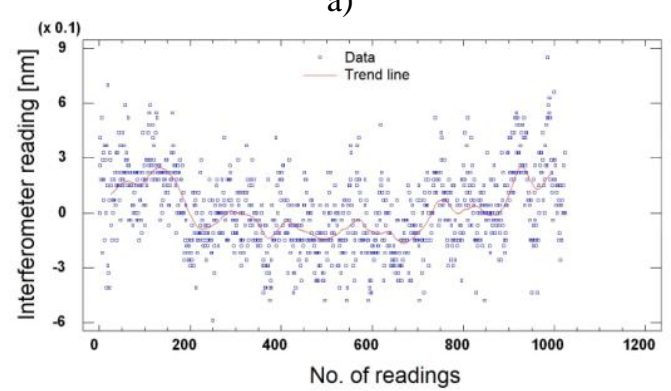

b)

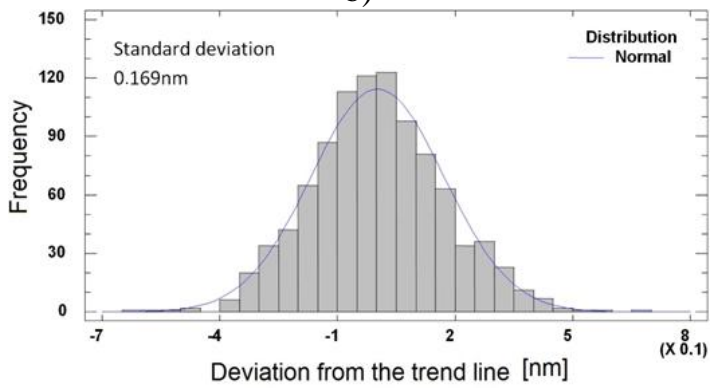

Fig. 6. a) Exemplary series of 1024 readings of the same measuring reflector position placed $1 \mathrm{~mm}$ from the zero value of the OPD. b) Histogram of differences between interferometer readings and the trend line.

a)

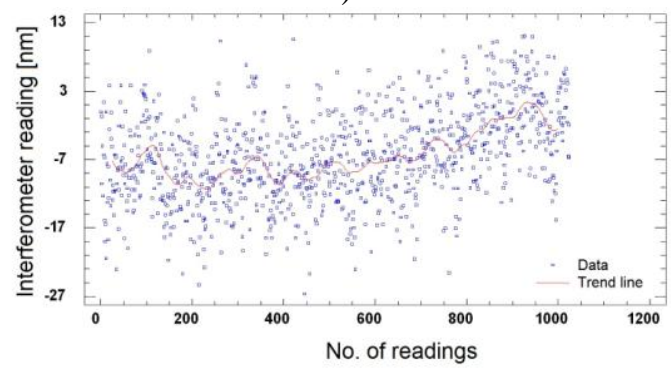

b)

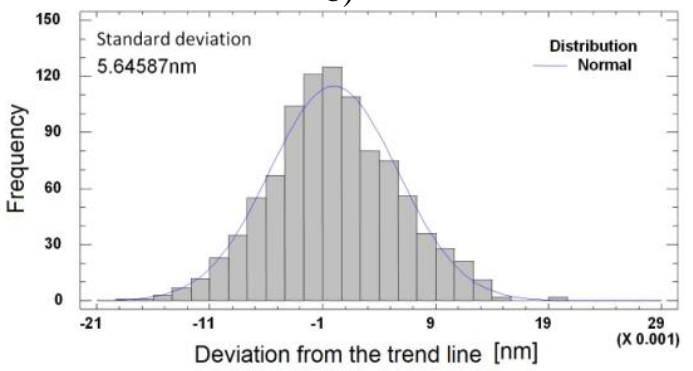

Fig. 7. a) Exemplary series of 1024 readings of the same measuring reflector position placed $1.5 \mathrm{~m}$ from the zero value of the OPD. b) Histogram of differences between interferometer readings and the trend line.

a)

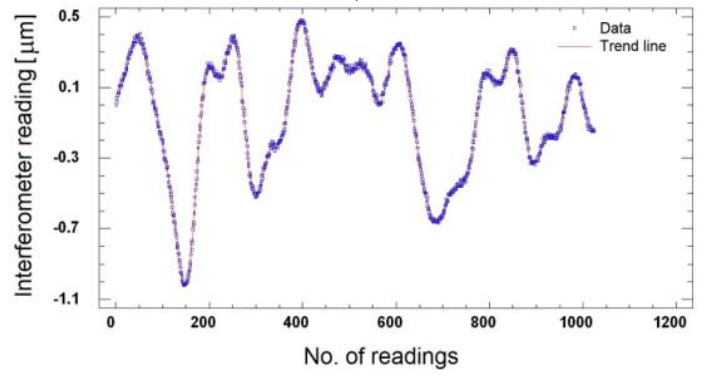

b)

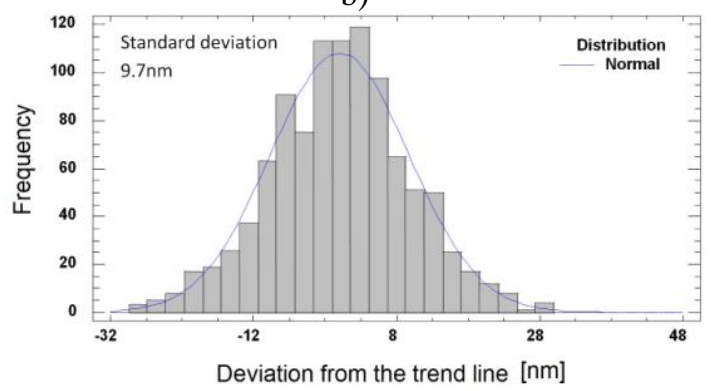

Fig. 8. a) Exemplary series of 1024 readings of the same measuring reflector position placed $2.5 \mathrm{~m}$ from the zero value of the OPD. b) Histogram of differences between interferometer readings and the trend line.

a)

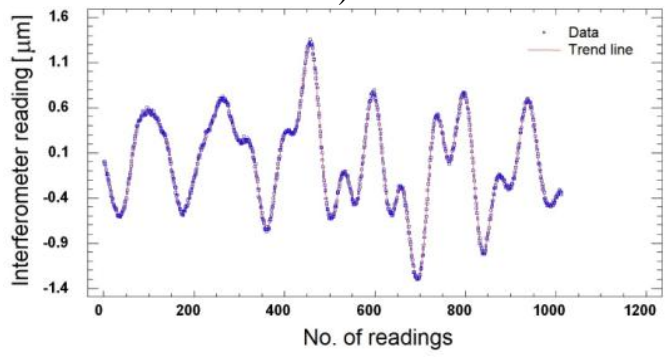

b)

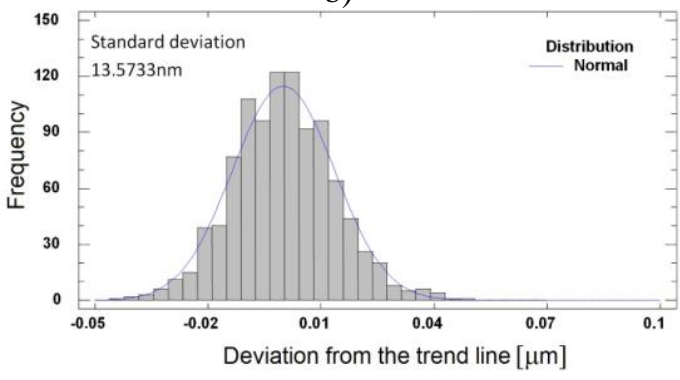

Fig. 9. a) Exemplary series of 1024 readings of the same measuring reflector position placed $3.5 \mathrm{~m}$ from the zero value of the OPD. b) Histogram of differences between interferometer readings and the trend line. 
Despite this discretization noise the overall probability distribution of the readings is still normal. Particularly Figs 9 and 10 show significant visible changes in the average levels of interferometer readings. They were caused partly by the temperature instability of the ambient air as well as laser diode emitted wavelength instability. However the main factor in this case was mechanical instability of the interferometer because for these distances the measuring reflector was placed on a separate table than the beamsplitter. Expanded uncertainties of the reflector position evaluation (basing on residuals between readings and the trend line) were calculated using a coverage factor $\mathrm{k}=2$ [24]. Obtained values of these uncertainties are equal to $\pm 0.3 \mathrm{~nm}, \pm 11.3 \mathrm{~nm}, \pm 19.4 \mathrm{~nm}$, and $\pm 27.1 \mathrm{~nm}$, for reflector distances equal to $1 \mathrm{~mm}, 1.5 \mathrm{~m}$, $2.5 \mathrm{~m}$ and $3.5 \mathrm{~m}$ respectively. The relevant range of the results is equal to $1 \mathrm{~nm}, 35 \mathrm{~nm}, 60 \mathrm{~nm}$ and $100 \mathrm{~nm}$. The experiment, which summarizes the dependence described above is shown in Fig. 10 .

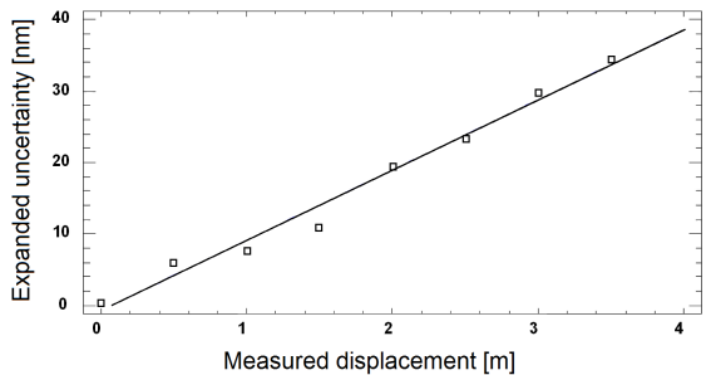

Fig. 10. Expanded uncertainty of the reflector position evaluation (basing on residuals between readings and the trend line) calculated using a coverage factor $\mathrm{k}=2$ versus measured displacement (equal to half of the OPD) of the interferometer.

Squares denote the results obtained from the experiment. The continuous line represents the linear regression model. The observed slope coefficient of the regression line is equal to about $10 \mathrm{~nm} / \mathrm{m}$. Basing on this model the expanded uncertainty of the interferometer single reading is equal to $\pm(0.7+10 \bullet l)[\mathrm{nm}]$, were $l$ is the measured distance in meters. The observed significant growth of the uncertainty is caused by two factors: (i) limited coherence length of the laser and (ii) LD injection current noise.

\section{Conclusions}

A novel laser diode based length measuring interferometer for scientific and industrial measurements has been presented. The applied wavelength stabilization system is based on the optical wedge interferometer.

Main components of the interferometer such as: laser diode stabilization assembly, photodetection system, measuring software, air parameters compensator, and optical assemblies have been described. Novel ideas and techniques applied in this components design have been shown. Metrological properties of the device such as resolution, measuring range, repeatability and accuracy were verified experimentally. Resulting from these studies full characteristics of the developed interferometer are summarized in Table 3.

The metrological specifications of the developed LD interferometer presented in Table 3 are in several cases significantly worse than those offered by commercially available $\mathrm{HeNe}$ laser based interferometers. However the obtained metrological and operational parameters are sufficient to many metrological, especially industrial, applications. The main advantages of the developed system are: small dimensions, high resistance to external magnetic fields, long lifetime of the applied type of laser $(100000 \mathrm{~h})$ and relatively low-cost construction. 
Table 3. Basic specifications of the developed LD interferometer.

\begin{tabular}{|c|c|c|}
\hline Parameter & $\begin{array}{l}\text { Experimental } \\
\text { value }\end{array}$ & Comments \\
\hline Wavelength & $635 \pm 10 \mathrm{~nm}$ & $\begin{array}{l}\text { Tolerance resulting from the differences } \\
\text { between individual items of lasers }\end{array}$ \\
\hline Frequency stabilization technique & \multicolumn{2}{|c|}{ Optical wedge interferometer } \\
\hline $\begin{array}{l}\text { Measuring range of: } \\
\begin{aligned}- & \text { linear displacement } \\
- & \text { angular displacement }\end{aligned}\end{array}$ & $\begin{array}{c}\text { From } 2 \text { to } 3.5 \mathrm{~m} \\
\pm 5^{\circ}\end{array}$ & $\begin{array}{l}\text { Depends on HL6312G laser item and } \\
\text { point of operation. Full range - for } \\
\text { Ondax } 635 \mathrm{LD} \text {. }\end{array}$ \\
\hline $\begin{array}{l}\text { Measurement resolution of: } \\
\begin{array}{c}\text { - } \\
\text { - }\end{array} \text { angular displacement }\end{array}$ & $\begin{array}{l}\approx 0.3 \mathrm{~nm} \\
\approx 0.5^{\prime \prime}\end{array}$ & Depends on OPD \\
\hline $\begin{array}{l}\text { Expanded uncertainty of singular } \\
\text { reading (coverage factor } \mathrm{k}=2 \text { ) }\end{array}$ & $\begin{array}{c} \pm(0.7+10 * l) \\
{[\mathrm{nm}]}\end{array}$ & $\begin{array}{l}\text { This value do not include influence of } \\
\text { air conditions. } l-m^{2} \text { measured } \\
\text { displacement in }[\mathrm{m}]\end{array}$ \\
\hline $\begin{array}{l}\text { Relative wavelength instability in } \\
\text { vacuum (during } 8 \mathrm{~h} \text { period of time) }\end{array}$ & $\begin{aligned} & 5 \times 10^{-9} \\
\approx & 5^{*} 10^{-8}\end{aligned}$ & $\begin{array}{l}\text { - Allan deviation (aver. time 1s) } \\
\text { - Relative average deviation }\end{array}$ \\
\hline Wavelength reproducibility & $\approx 5 \times 10^{-8}$ & (in vacuum) \\
\hline $\begin{array}{l}\text { Relative inaccuracy of meas. } \\
\begin{aligned}- & \text { Linear displacement } \\
- & \text { Angular displacement }\end{aligned}\end{array}$ & $\begin{aligned} & \approx 10^{-6} \\
\approx & 1.5 \times 10^{-4}\end{aligned}$ & Including influence of air conditions. \\
\hline Laser beam diameter & $4 \mathrm{~mm}$ & \\
\hline $\begin{array}{l}\text { Distance between the outgoing and } \\
\text { the returning beams }\end{array}$ & $10 \mathrm{~mm}$ & \\
\hline Stabilization time & $\approx 25 \mathrm{~min}$ & \\
\hline Temperature range of operations & $\begin{array}{l}\text { from } 15^{\circ} \mathrm{C} \text { to } \\
30^{\circ} \mathrm{C}\end{array}$ & \\
\hline $\begin{array}{l}\text { Maximum measuring reflector } \\
\text { velocity }\end{array}$ & $1.6 \mathrm{~m} / \mathrm{s}$ & $\begin{array}{l}\text { Results from the current design of the } \\
\text { interpolator }\end{array}$ \\
\hline Laser head dimensions & $61 \times 71 \times 110 \mathrm{~mm}$ & \\
\hline
\end{tabular}

\section{Acknowledgements}

This work was supported by the German Ministry of Science and Higher Education under contract No. R01 011 02. The author thanks all contractors of this project for their contribution to the development of the laser diode interferometer described above.

\section{References}

[1] Atkinson, L.G. (1994). Compact distance measuring interferometer. U.S. Patent No. 5374991.

[2] Takaaki, H., Mamoru, H., Hideki, N. (2001). Wavelength stable laser diode and photodiode array for laser interferometer positioning systems. Yokogava Technical Report English Edition, 32, 1-4.

[3] Dudzik, G. (2009). Laser frequency standard 780nm/87Rb with Zeman splitting of atomic line. PhD dissertation. (in Polish)

[4] Budzyn, G., Dudzik, G., Rzepka J. (2007). Frequency Stabilization of a Single Mode 780nm VCSEL Lasers with the Use of a Rubidium Cell. In ICTON-MW'07 conf. proc., 1-3.

[5] Donati, S., Falzoni, L., Merlo, S. (1996). A PC-Interfaced, compact laser diode feedback interferometer for displacement measurements. IEEE Transactions on Instrumentation and Measurement, 45(6), 942-947.

[6] Sasaki, O., Yoshida, T., Suzuki, T. (1991). Double sinusoidal phase-modulating laser diode interferometer for distance measurement. Applied Optics, 30, 3617-3621. 
[7] Wang, X., Wang, X., Lu, H., Qian, F., Bu, Y. (2001). Laser diode interferometer used for measuring displacements in large range with a nanometer accuracy. Optics and Laser Technology, 33, 219-223.

[8] Ishii, Y., Takahashi, T. (2002). Laser-diode phase-conjugate interferometer for distance measurement. Journal of Optics A: Pure and applied optics., 4 , S327-S331.

[9] UPM miniature laser interferometer. (2005). Reitz-micro-contact - Reitz-motion-control.

[10] Hoogeveen, T. (2003). Stabilizing a diode laser to an external reference. Development of the electronic, $1-53$.

[11] De Labachelerie, M., Latrasse, C., Kemssu, P., Cerez, P. (1992). The frequency control of laser diodes. $J$. Phys. tit France 2, 1557-89.

[12] Fox, R.W., Zibrov, A.S., Hollberg, L. (1997). Semiconductor Diode Lasers. In Experimental Methods in the Physical Sciences, 29C, 77-102.

[13] Kruger, J.M.W. (1998). A Novel Technique for Frequency Stabilising Laser Diodes. DipSci Thesis, 1-39.

[14] Gawlik, W., Zachorowski, J. (2004). Stabilization of diode-laser frequency to atomic transitions. Opt. Applicata, 34, 4.

[15] Zhmud, A.A. (2001). Differential Method of Diode Laser Wavelength Stabilization. Jpn. J. Appl. Phys., 40, 5947-48.

[16] Brasunas, J.C.(2002). A simple etalon-stabilized visible laser diode Meas. Sci. Technol. (Design Note). 13, N67-N71.

[17] Dobosz, M. et all. (2011). Laser diode based interferometric length measurement system. Project of Ministry of Science and Higher Education No. R01 01102 report. (in Polish)

[18] Dobosz, M., Kożuchowski, M. (2012). Frequency stabilization of a laser diode by means of an optical wedge etalon. Measurement Science and Technology, 23, 1-9.

[19] Allan, D.W. (1966). Statistics of atomic frequency standards. In Proc. IEEE., 54, 221-30.

[20] Dobosz, M., Zamiela, G. (2012). Interference fringe detection system for distance measuring interferometer. Optics and Laser Technology, 44, 1620-1628.

[21] Dobosz, M., Zamiela, G. (2009). Method of light flux changes processing into two electrical signals and laser interferometer system to use this way. Patent application P-387342. (in Polish)

[22] Dobosz, M., Zamiela, G. (2009). Interferometer for measuring linear and angular displacements. Patent application P-387343. (in Polish)

[23] Maxim Dallas Semiconductor Application Note 208. Curve fitting the Error of Bandgap-Based Digital Temperature Sensor (www.maxim-ic.com).

[24] EA-4/02. (2002). Expression of the uncertainty of measurement in calibration. 